DOI: http://dx.doi.org/10.18203/2320-1770.ijrcog20183288

Review Article

\title{
Cancer in pregnancy
}

\section{Reddi Rani P., Ashwini Vishalakshi L.*}

Department of Obstetrics and Gynecology, Mahatma Gandhi Medical College and Research Institute, Sri Balaji Vidyapeeth University, Pilliyarkuppam, Pondicherry, Tamil Nadu, India

Received: 28 May 2018

Accepted: 26 June 2018

\section{* Correspondence:}

Dr. Ashwini Vishalakshi L.,

E-mail: akshivishal@gmail.com

Copyright: (c) the author(s), publisher and licensee Medip Academy. This is an open-access article distributed under the terms of the Creative Commons Attribution Non-Commercial License, which permits unrestricted non-commercial use, distribution, and reproduction in any medium, provided the original work is properly cited.

\begin{abstract}
Cancer in pregnancy though rare is a challenging problem both for patient and obstetrician. It is on rise though pregnancy does not predispose cancer. Persistent symptoms like vomiting, abdominal pain, discharge etc should be thoroughly evaluated by clinical USG and if necessary by MRI if there is a strong suspicion of malignancy to detect early and treat promptly. A multidisciplinary discussion is necessary. Management depends on type of malignancy, stage, risk factors, histopathology and gestational age. Carcinoma of breast and cervix are common malignancies that occur in pregnancy. It is preferable to postpone surgery and CT till first trimester is completed. A through counseling is essential regarding termination of pregnancy, type of treatment and its effects in mother and fetus and prognosis. Aim should be curative treatment to the mother with some modifications in the treatment keeping in mind maternal and fetal safety.
\end{abstract}

Keywords: Cancer, Chemotherapy, Pregnancy, Radiotherapy, Surgery

\section{INTRODUCTION}

Cancer during pregnancy is a special challenge as cancer or its treatment may affect not only the pregnant woman but also fetus and needs proper counseling and multidisciplinary approach. It is the second most common cause of death in women during their reproductive age and complications between $0.02 \%-0.1 \%$ of all pregnancies. ${ }^{1}$ The incidence is increasing due to concomitant increase in the age of child bearing. The most common cancers seen during pregnancy are breast and cervical carcinomas.

Others which are rarely found are lymphomas, Hodgkin's disease, malignant melanoma, ovarian tumors especially germ cell and borderline epithelial tumors etc. Curative treatment of the mother is the main priority and should adhere to standard treatment protocols with some modifications without causing harm to the fetus. Ethical, personal and emotional factors vary between patients and makes management difficult.

\section{DIAGNOSIS DURING PREGNANCY}

Cancer diagnosis in pregnancy poses a very difficult challenge to woman, her family and doctor. The benefits of diagnostic workup, treatment modalities like surgery, radiotherapy, and chemotherapy (CT) has to be weighed carefully against the risks to the fetus.

Diagnosis may be delayed as symptoms of pregnancy like nausea, vomiting, breast changes, abdominal pain, anemia, bleeding or vaginal discharge all these physiological changes may mimic malignancy symptoms.

So careful clinical examination is necessary for persisting or worsening symptoms, so that delay in diagnosis and treatment can be avoided. Clinical examination also may be difficult due to gravid uterus and breast changes. 
Imaging techniques are restricted to ultrasound and MRI and also there is a decrease in utility of tumor markers. All these factors are contributing for delay in diagnosis and also there is hesitance in starting antineoplastic treatment in pregnancy. There is a need to weigh the risks and benefits for both maternal and fetal wellbeing.

\section{STAGING OF CANCER IN PREGNANCY}

It should be comprehensive staging using clinical and imaging modalities like ultrasound and MRI and also avoiding CT scan and radiographic examination. If deemed necessary radiation exposure should be as low as reasonably achievable to avoid detrimental effects on the fetus. A dose of $100 \mathrm{mGy}$ has detrimental effects on the fetus causing lethality, malformations and mental retardation. ${ }^{2}$ For pathological confirmation tissue biopsy is more accurate than fine needle aspiration cytology. Reporting pathologist has to keep in mind the physiological hyperproliferative changes while reporting. ${ }^{3}$

\section{GENERAL PRINCIPLES OF TREATMENT}

After diagnosis of cancer patient and relatives should be counseled regarding decision making, explaining her about her stage of disease, effects of treatment on mother and fetus, regarding pregnancy termination, iatrogenic prematurity or intentional delay in treatment and prognosis. Treatment should be individualized and should be counseled regarding the risk of recurrence with future pregnancies and need for continuous surveillance. The modalities of treatments are surgery, radiotherapy (RT), chemotherapy (CT), usually combinations of two or three modalities in advanced stages.

\section{SURGERY}

Though it can be done in all trimesters, avoid if possible during first trimester. Some modifications have to be done in anesthesia and surgery without compromising the curative treatment and considering the fetal and maternal safety and prevention of obstetrical complications like abortion and preterm labor. Surgery depends on the age of the patient, gestational age of pregnancy, type of malignancy, stage of disease and histopathology report.

\section{PREOPERATIVE PREPARATION}

A proper preoperative oxygenation, antacids and premedication are necessary to minimize aspiration pneumonitis and anxiety. Antibiotics prophylactic/ therapeutic doses should be given depending on the specific surgical procedure. Antibiotics should be chosen which are safe in pregnancy and lactation. They are at high risk for thromboembolism due to pregnancy, malignancy, surgery and immobilization and should be advised thromboprophylaxis with unfractionated or low molecular weight heparin.
Fetal anoxia can occur during surgery due to maternal hypotension, deep general anesthesia, hypovolemia and venacaval compression. Fetal oxygenation during surgery is very important. Venacaval compression can be avoided by left lateral tilt and maintaining $\mathrm{PaO} 2$ and $\mathrm{PaCO} 2$ and uterine blood flow. A stable maternal condition is the best guarantee for fetal wellbeing. One of the earliest signs of maternal distress is fetal distress. ${ }^{4}$ There is a need for continuous cardiotocographic monitoring during surgery especially if she is in the third trimester and if there is any abnormality caesarean section can be done at the same time.

Tocolytics are indicated to prevent abortion and preterm labor especially in abdominal surgery where there is a possibility of uterine manipulation. Adequate analgesia is required postoperatively. Surgery maybe diagnostic or therapeutic which may be associated with prematurity either due to surgical procedure or due to exposure to anesthesia. $^{5}$ In case of laparotomy midline vertical incision is preferred for optimal exposure. Laparoscopic approach is preferred if feasible during pregnancy depending on the size of uterus and modification has to be done for safe entry into abdomen. ${ }^{6}$ Laparoscopy becomes technically difficult after 26-28 weeks of gestation due to gravid uterus and in these cases laparotomy is preferred. ${ }^{7}$ Expert consensus meeting recommended the prerequisites for treating malignancy during pregnancy with laparoscopy are; a maximal laparoscopic procedure time of 90 minutes, a pneumoperitoneum with a maximum intra-abdominal pressure of $10-13 \mathrm{mmHg}$, open introduction and experienced surgeon. ${ }^{8}$

\section{RADIOTHERAPY DURING PREGNANCY}

Radiotherapy during pregnancy to cancers other than genital tract malignancies is safe when pregnancy is still early, and adequate distance exists between radiation field and the fetus and fetal radiation dose is less than 100 mGy. Treatment plan has to be modified by changing field angles, reducing field size, modifying the beam energy etc. It is important to calculate the fetal dose by measurements in a phantom before treatment is given. Montecarlo methodology can be used to evaluate and estimate fetal dose. ${ }^{9}$ Long-term follow up of children exposed to radiation in uterus suggests that RT to upper body parts before third trimester and with shielding of pregnancy does not induce fetal harm.

\section{CHEMOTHERAPY DURING PREGNANCY}

Chemotherapy during first trimester of pregnancy may increase the risk of spontaneous abortions, fetal death and major congenital malformations. The teratogenic effects depend on type of chemotherapeutic agent, dosage, gestational age at administration and cumulative effect. The most vulnerable period is $2-8$ weeks when organogenesis occurs. First trimester exposure has been associated with $10-20 \%$ risk of major malformation 
which can reduce to about $6 \%$ when folate antagonists are excluded. ${ }^{10}$

Cardonic et al in a review of 376 cases of fetuses exposed to CT in utero after organogenesis demonstrated $5 \%$ fetal death, $1 \%$ neonatal death, 5\% preterm delivery, $7 \%$ IUGR and $4 \%$ transient myelosuppression. ${ }^{11}$

Administration of CT during second and third trimester of pregnancy is associated with major congenital malformations but may be associated with increased risk of IUGR, preterm and stillbirths. CT is advised only after 12-14 weeks of pregnancy because of teratogenicity risks and also by then placenta develops which acts as a barrier and protects the fetus. Administration of CT during pregnancy is not an indication for termination of pregnancy and it allows timely maternal treatment without delay and helps in prevention of iatrogenic prematurity.

\section{Alkylating agents}

Cyclophosphomide is commonly used for the treatment of breast cancer, ovarian cancer and non hodgkins lymphoma which act directly on cell DNA to prevent rapidly replicating cells from reproducing. In an analysis of outcome of 61 patients treated for different malignancies during second and third trimester found 59 infants were born with no malformations. ${ }^{12}$

\section{Platinum compounds}

Cisplatin and carboplatin are the most commonly used drugs in carcinoma cervix, ovarian cancer, lung cancer etc. Their use in second and third trimester was not associated with adverse fetal effects.

\section{Antimetabolites}

Methotrexate, 5-flurouracil, mercaptopurine etc. come under this category and cause inhibition of cellular metabolism. Methotrexate exposure during first trimester can cause malformations like cranial dysostosis, hypertelorism, wide nasal bridge, micrognathia etc. ${ }^{13}$

\section{Anthracyclines}

These include doxorubicin, epirubicin ect. Cardmock et al observed pregnancy outcome in 118 patients treated for breast cancer and lymphoma during second and third trimester and found five abnormal fetuses with anomalies. ${ }^{11}$ Plant alkaloids like taxanes are also safe during second and third trimester.

Safety of molecularly targeted agents use like tyrosine kinase inhibitors, monoclonal antibody, rituximab etc. has not been established in pregnancy.

Timing of delivery should be planned to avoid myelosupression. It should be stopped 2-3 weeks before delivery or pregnancy continued 2-3 weeks following anticancer treatment if it is feasible for bone marrow recovery. ${ }^{14}$ As cancer chemotherapeutic drugs are excreted into milk, they should be avoided during lactation or if it is essential to give CT avoid breastfeeding.

\section{SOGC GUIDELINES FOR CT IN PREGNANCY}

1. Most of the women in childbearing age are postponing their pregnancy, so more cases are being diagnosed with cancer in pregnancy. Doctor should examine the patient's risk of pregnancy and desire to prevent pregnancy during CT.

2. CT agents used to combat cancer cases cross the placenta and may adversely affect embryogenesis by affecting cell division and results in major congenital malformations. Exposure to CT drugs after first trimester is not associated with increased risk of malformations but with increased risk of stillbirths IUGR and fetal toxicity.

3. Decision about the best course of management in pregnancy including timing of delivery should balance maternal and fetal risks.

4. They should be optimally managed by a multidisciplinary team. ${ }^{15}$

\section{OBSTETRIC AND PERINATAL MANAGEMENT}

Initial ultrasound at booking should be done to evaluate the fetal gestational age, anomalies, later for fetal growth and development by regular fetal monitoring. Cervical length should be measured especially in cases of early carcinoma cervix where cone biopsy/ radical trachelectomy was done to assess for cervical incompetence. If $\mathrm{CT}$ is ongoing delivery should be planned 3 weeks after the last cycle of CT during pregnancy to avoid accumulation in the neonate and also to avoid problems associated with hematopoietic suppression during delivery and for the same reason CT should not be administered after 35-37 weeks since spontaneous labor can occur any time.

Mode of delivery is vaginal in majority of cases except in carcinoma cervix and where there is metastatic disease in long bones where there may be a risk of fracture due to lithotomy position. It is also indicated in cases where there is CNS metastasis causing increased intracranial pressure due to active pushing in vaginal delivery. ${ }^{8}$ Caesarean section is also indicated in cases of vulval cancer where there is extensive scarring and vulval trauma.

Rarely placental metastasis has been reported. Proven metastasis to the fetus is rare. Malignancies spreading to products of conception are melanoma (32\%), leukemia and lymphoma $(5 \%)$, breast cancer $(13 \%)$, lung cancer (11\%), gynecological cancer $(3 \%)$ etc. ${ }^{16}$ In those having placental metastases the infant should be monitored for development of malignant disease. Breast feeding is 
allowed unless CT is ongoing postpartum or administered in the last week before delivery.

\section{LONG TERM FOLLOW-UP OF CHILDREN}

Recent evidence suggests CT is safe beyond first trimester. Aviles et al reported in a follow up of mean 18.7 yrs of 84 children born to mothers of hematological malignancies who received $\mathrm{CT}$ during pregnancy and found no congenital neurological or psychological abnormalities and they had good learning and educational performances. ${ }^{17}$ Another concern is the potential effect of anthracyclines on the fetal heart. These are commonly used in breast and hematological cancers. Aviles et al reported follow up of 81 children exposed to cytotoxic drugs including anthracyclines in utero found no evidence of cardiac disease and echo and fractional shortening were normal. ${ }^{18}$

\section{CONCLUSION}

Carcinoma in pregnancy is rare. Management is a challenge as it is concerned about mother and fetus. Treatment should be multidisciplinary. Optimizing the treatment of cancer and minimizing harm to the fetus are the main goals which depend on stage of disease and gestational age. Treatment has to be individualized depending on the type of cancer, stage, histopathology and gestational age. Modalities of treatment include surgery CT and RT. Surgery and CT are better preferred after first trimester ultrasound necessary to exclude congenital anomalies and also to monitor fetal growth as it may be associated with IUGR. Continuation of pregnancy till 35- 37 weeks helps to prevent neonatal and long term cognitive problems induced by preterm birth. The pros and cons of continuing and terminating the pregnancy should be weighed from the physical and psychological wellbeing.

\section{Funding: No funding sources}

Conflict of interest: None declared

Ethical approval: Not required

\section{REFERENCES}

1. Lishner M. Cancer in pregnancy. Ann Oncol. 2007;14(supplement 3):1-6.

2. Stovall M, Blackwell CR, Cundiff J, Novack DH, Palta JR, Wagner LK, et al. Fetal dose from radiotherapy with photon beams: report of AAPM Radiation Therapy Committee Task Group No. 36. Med Physics. 1995;22(1):63-82.

3. Van Calsteren K, Vergote I, Amant F. Cervical neoplasia during pregnancy: diagnosis, management and prognosis. Best Practice Res Clin Obstet Gynaecol. 2005;19(4):611-30.

4. Evans SR, Sarani B, Bhanot P et al. Surgery in pregnancy. Curr Probl Surg. 2012;49:333-8.
5. Cohen-Kerem R, Railton C, Oren D, Lishner M, Koren G. Pregnancy outcome following nonobstetric surgical intervention. Am J Surgery. 2005;190(3):467-73.

6. Yumi H. Guidelines for diagnosis, treatment, and use of laparoscopy for surgical problems during pregnancy. Surg Endosc. 2008;22(4):849-61.

7. Pearl J, Price R, Richardson W, Fanelli R. Guidelines for diagnosis, treatment, and use of laparoscopy for surgical problems during pregnancy. Surg Endosc. 2011;25(11):3479.

8. Amant F, Halaska MJ, Fumagalli M, Steffensen KD, Lok C, Van Calsteren K, et al. Gynecologic cancers in pregnancy: guidelines of a second international consensus meeting. Int $\mathrm{J}$ Gynecol Cancer. 2014;24(3):394-403.

9. Bednarz B, Xu XG. A feasibility study to calculate unshielded fetal doses to pregnant patients in 6-MV photon treatments using Monte Carlo methods and anatomically realistic phantoms. Med Physics. 2008;35(7 Part1):3054-61.

10. Doll DC, Ringenberg QS, Yarbro JW. Antineoplastic agents and pregnancy. In Seminars Oncol. 1989;16(5):337-46.

11. Cardonick E, Iacobucci A. Use of chemotherapy during human pregnancy. Lancet Oncol. 2004;5(5):283-91.

12. Vancalastern K, Heyns L, Smet FD, Eycken V, Gziri MM, Halaska M, et al. Cancer during pregnancy: an analysis of 215 patients emphasizing the obstetrical and neonatal outcome. J Clin Oncol. 2010;28:683-9.

13. Powell HR, Ekert H. Methotrexate-induced congenital malformations. Med J Australia. 1971;2(21):1076.

14. Pereg D, Koren G, Lishner M. Cancer in pregnancy: gaps, challenges and solutions. Cancer Treatment Rev. 2008;34(4):302-12.

15. Koren G, Carry N, Gagon R, Maxwell C, Nulman I, Senikas V. Cancer chemotherapy and pregnancy. Send to J Obstet Gynaecol Can. 2013;35(3):263-278

16. Alexander A, Samlowski WF, Grossman D, Bruggers CS, Harris RM, Zone JJ, Noyes RD, et al. Metastatic melanoma in pregnancy: risk of placental metastases in the infant. J Clin Oncol. 2003;21(11):2179-86

17. Avilés A, Neri N. Hematological malignancies and pregnancy: a final report of 84 children who received chemotherapy in utero. Clinical Lymphoma. 2001;2(3):173-7.

18. Aviles A, Neri N, Nambo MJ. Long-term evaluation of cardiac function in children who received anthracyclines during pregnancy. Ann Oncol. 2005;17(2):286-8.

Cite this article as: Reddi RP, Vishalakshi AL. Cancer in pregnancy. Int J Reprod Contracept Obstet Gynecol 2018;7:2989-92. 